\title{
OCORRÊNCIA DE LARVAS DE SCARABAEIDAE FITÓFAGOS (INSECTA: COLEOPTERA) EM DIFERENTES SISTEMAS DE SUCESSÃO DE CULTURAS ${ }^{1}$
}

\author{
Sérgio Roberto Rodrigues ${ }^{2}$, José Ivaldo do Carmo ${ }^{2}$, \\ Vilma dos Santos Oliveira ${ }^{2}$, Elison Floriano Tiago ${ }^{2}$, Tiago Ledesma Taira ${ }^{2}$
}

\section{ABSTRACT \\ OCCURRENCE OF PHYTOPHAGOUS SCARABAEIDAE \\ (INSECTA: COLEOPTERA) LARVAE IN DIFFERENT SUCCESSION CROP SYSTEMS}

Information about Scarabaeidae phytophagous are still rare for the Center-West Region of Brazil. Thus, in the experimental area of the Universidade Estadual do Mato Grosso do Sul, in Aquidauana, Mato Grosso do Sul State, Brazil, the succession of soybean, maize, and forage turnip was sowed from February 2004 to October 2005, when the larvae population dynamics was evaluated. From October 2006 to May 2008, nine succession systems were sowed, the larval density evaluated, and the identification of the current species was carried out. When the larvae were sampled for soybean, in January 2005 and 2006, the highest densities were found in the field (3.44 larvae $\mathrm{m}^{-2}$ and 4.19 larvae $\mathrm{m}^{-2}$, respectively). The forage turnip, sampled in October 2004 and 2005, showed the lowest densities ( 0.03 larvae $\mathrm{m}^{-2}$ and 0.02 larvae $\mathrm{m}^{-2}$, respectively). In the crop succession systems, the following species were found: Liogenys fuscus Blanchard (highest amount), Liogenys bidenticeps Moser, Anomala testaceipennis Blanchard, Paranomala inconstans (Burmeister), Geniates borelli Camerano, Cyclocephala forsteri Endrodi, Cyclocephala verticalis Burmeister, and Phyllophaga sp. For the succession systems with maize sowed at the traditional season, the highest larval densities were found, specially favoring the development of L. fuscus, while the succession systems with soybean, maize, and under fallow, and soybean, crotalaria, and forage turnip, as well as the three systems with cotton-plant, did not favor it.

KEY-WORDS: Zea mays; Glycine max; Raphanus sativus; soil pests; larvae density.

\section{INTRODUÇÃO}

Larvas de Scarabaeidae fitófagos são consideradas pragas importantes em várias culturas, pois, ao se alimentarem do sistema radicular, causam sérias injúrias às plantas. Além disto, infestações mistas de diferentes espécies são, normalmente, encontradas nas lavouras, o que pode dificultar a aplicação de

\section{RESUMO}

Informações sobre Scarabaeidae fitófagos são ainda escassas para a região Centro-Oeste do Brasil. Por esta razão, na área experimental da Universidade Estadual do Mato Grosso do Sul, em Aquidauana (MS), foi semeada, de fevereiro de 2004 a outubro de 2005, a sucessão de culturas soja, milho e nabo forrageiro, onde se avaliou a dinâmica da densidade larval. De outubro de 2006 a maio de 2008, foram realizadas semeaduras de nove sistemas de sucessão de culturas, sendo avaliadas as densidades larvais e identificadas as espécies ocorrentes. Quando se amostraram as larvas na cultura da soja, em janeiro de 2005 e 2006, encontraram-se as maiores densidades $\left(3,44\right.$ larvas $\mathrm{m}^{-2} \mathrm{e}$ 4,19 larvas $\mathrm{m}^{-2}$, respectivamente), no campo. O nabo forrageiro, amostrado em outubro de 2004 e 2005, apresentou as menores densidades ( 0,03 larvas $\mathrm{m}^{-2}$ e 0,02 larvas $\mathrm{m}^{-2}$, respectivamente). Nos sistemas de sucessão de culturas, as espécies encontradas foram Liogenys fuscus Blanchard, Liogenys bidenticeps Moser, Anomala testaceipennis Blanchard, Paranomala inconstans (Burmeister), Geniates borelli Camerano, Cyclocephala forsteri Endrodi, Cyclocephala verticalis Burmeister e Phyllophaga sp, sendo que L. fuscus foi coletada em maior quantidade. Para os sistemas de sucessão que possuem o milho semeado na safra, foram encontradas as maiores densidades larvais, indicando que esta cultura favorece o desenvolvimento, principalmente, de L. fuscus, enquanto os sistemas de sucessão com soja, milho e pousio, e soja, crotalária e nabo forrageiro, bem como os três sistemas com o algodoeiro, lhe são desfavoráveis.

PALAVRAS-CHAVE: Zea mays; Glycine max; Raphanus sativus; pragas do solo; densidade de larvas.

algumas táticas de controle, como o uso de inseticidas (Pérez-Agis et al. 2008), aumentando, assim, a importância dos estudos sobre este complexo de pragas.

Desde meados da década de 1980, um complexo de larvas de Scarabaeidae tem causado danos à cultura da soja, em várias regiões do Brasil (Oliveira \& Garcia 2003), sendo Phyllophaga cuyabana Moser a principal espécie, no Estado do Pa-

1. Trabalho recebido em out./2009 e aceito para publicação em jan./2011 (nº registro: PAT 7698/ DOI 10.5216/pat.v41i1.7698).

2. Universidade Estadual do Mato Grosso do Sul, Faculdade de Agronomia, Departamento de Entomologia, Aquidauana, MS, Brasil.E-mails: sergio@uems.br, ze.agro2009@hotmail.com, vilma.agro@hotmail.com, elisonfloriano@yahoo.com, 
raná. Já no Rio Grande do Sul, no final da década de 1980, em cultura de inverno, larvas de Scarabaeidae se destacaram pelos danos ocasionados, sendo identificada Diloboderus abderus (Sturm) como a principal espécie ocorrente (Silva \& Costa 2002).

Após a identificação destas espécies de pragas, no Sul do Brasil, estudos sobre aspectos biológicos (Oliveira et al. 1996, Silva \& Loeck 1996), nível de controle (Silva 1997, Silva \& Costa 2002) e técnicas para diminuição de densidades larvais (Silva et al. 1994, Oliveira et al. 2000) foram conduzidos. Entretanto, pesquisas sobre estas pragas são ainda insuficientes para outras regiões do País, como o Centro-Oeste, principalmente devido ao complexo de espécies de Scarabaeidae fitófagos ser diferente do encontrado na Região Sul.

Em Mato Grosso do Sul, Santos \& Ávila (2007, 2009) estudaram os aspectos bioecológicos de Cyclocephala forsteri Endrodi, amostrada em cultura de soja, e Liogenys suturalis Blanchard, em cultura de milho. Nesta região, Anomala testaceipennis Blanchard, Liogenys fuscus Blanchard e Cyclocephala verticalis Burmeister são consideradas importantes pragas, tendo sido realizados estudos sobre seus aspectos biológicos e ocorrência (Rodrigues et al. 2008ab, Rodrigues et al. 2010). No Distrito Federal e em Goiás, Oliveira et al. (2007, 2008) destacam Aegopsis bolboceridus (Thomson) como causadora de danos a hortaliças em ambiente protegido e em áreas cultivadas com milho, e P. capillata (Blanchard), em soja.

Para as várias regiões do Brasil, verifica-se que diferentes espécies de Scarabaeidae se destacam pela abundância e pelos danos proporcionados. Assim, torna-se importante conhecer, além das espécies presentes, a influência de algumas culturas utilizadas em sucessão sobre o complexo de Scarabaeidae no local, originando informações que possam auxiliar no controle deste grupo de pragas do solo, adaptadas para cada região.

Como para a região Centro-Oeste há poucas informações relacionadas aos Scarabaeidae fitófagos, foram desenvolvidos estudos, com o objetivo de se conhecer a dinâmica da densidade larval e o efeito da sucessão de culturas na densidade de larvas fitófagas de Scarabaeidae, na região de Aquidauana (MS).

\section{MATERIAL E MÉTODOS}

\section{Dinâmica da densidade larval}

Os trabalhos foram desenvolvidos na Fazenda Experimental da Universidade Estadual do Mato Grosso do Sul, no município de Aquidauana (MS), em uma área de dois hectares, anualmente cultivada sob sistema de plantio convencional. Nesta área, foi feita a semeadura de milho (Zea mays L.), em fevereiro de 2004, com a cultivar BR-106, sob sistema convencional.

As culturas instaladas após a retirada do milho foram semeadas sob sistema de plantio direto. Em julho e outubro de 2004, foram semeados nabo forrageiro (Raphanus sativus L.) e soja (Glycine max (L.)), respectivamente. Utilizou-se a cultivar de soja "BRSMS - mandi". Em 2005, foram implantadas as culturas de milho, nabo forrageiro e soja, em fevereiro, julho e outubro, respectivamente, sob sistema de plantio direto. Neste experimento, a sucessão soja-milho foi escolhida, por ser o sistema usualmente utilizado pelos agricultores em Mato Grosso do Sul.

Transcorridos de 80 a 90 dias da semeadura de cada cultura, foram realizadas amostragens de larvas de Scarabaeidae, coletando-se, ao acaso, 418 amostras de solo (25 cm x $25 \mathrm{~cm}$ x $30 \mathrm{~cm}$ de profundidade) por hectare, conforme metodologia adaptada de Silva \& Loeck (1996) e Silva \& Costa (1998). As amostras foram, então, peneiradas e as larvas de Scarabaeidae presentes quantificadas.

\section{Espécies de Scarabaeidae}

A identificação das espécies de Scarabaeidae presentes na área experimental, nas amostragens realizadas em janeiro e maio de 2008 , foi feita após os insetos completarem seu ciclo de vida, por meio do surgimento do besouro adulto. Para isto, 50 larvas (espécimes) foram mantidas vivas, em laboratório, e individualizadas em frascos plásticos de $500 \mathrm{~mL}$, contendo solo e mudas das plantas cultivadas, conforme adaptadações das metodologias descritas por Aragón-Garcia \& Morón (2000) e Pardo-Locarno et al. (2005). Com o emprego desta metodologia, ao se obterem os insetos adultos, pode-se providenciar a identificação das espécies e relacioná-las aos sistemas de sucessão de culturas.

Espécimes dos Scarabaeidae foram depositados na coleção "Adolph Hempel”, do Instituto Biológico, em São Paulo, e na coleção de insetos do Laboratório de Entomologia da Universidade Estadual do Mato Grosso do Sul, em Aquidauana (MS). 


\section{Efeito da sucessão de culturas na densidade de larvas}

Para realizar o estudo da sucessão de culturas sobre a densidade de larvas de Scarabaeaidae, foi instalado um experimento, na mesma área, a partir de outubro de 2006. Foram instalados nove sistemas de sucessão de culturas (Tabela 1), com quatro repetições, sendo cada repetição representada por uma parcela de $15 \mathrm{~m} \times 20 \mathrm{~m}$.

O experimento foi conduzido em delineamento de blocos casualizados, em esquema fatorial 9x6, utilizando-se nove sistemas de sucessão, com seis períodos de semeadura e quatro repetições. Os períodos de semeadura foram outubro de 2006; fevereiro, maio e outubro de 2007; e fevereiro e maio de 2008.

As culturas de soja, milho e algodão (Gossypium hirsutum L.) foram semeadas em outubro de 2006 e 2007. Na safrinha, as culturas de milho, girassol (Helianthus annuus L.) e as crotalárias e o nabo forrageiro foram semeados em fevereiro de 2007 e 2008. Em maio de 2007 e 2008, foi semeado o nabo forrageiro (Tabela 1 ).

Após transcorridos de 80 a 90 dias da semeadura, em cada cultura e nas parcelas em pousio, foram realizadas amostragens das larvas de Scarabaeidae ocorrentes. Para isto, 468 amostras de solo por hectare foram coletadas (as parcelas apresentavam $300 \mathrm{~m}^{2} \mathrm{de}$ área, onde foram realizadas 13 amostragens por parcela), para extração das larvas, da mesma maneira descrita para o estudo da dinâmica da densidade larval.

Os dados referentes às densidades de larvas apresentaram heterocedasticidade de variâncias, sendo transformadas em $\sqrt{x+1}$ e submetidas a análise de variância e as médias comparadas pelo teste Tukey, a 5\%. As análises foram realizadas pelo programa computacional ESTAT (Unesp 1994). Realizou-se análise de correlação (Pearson) entre as densidades médias larvais e os dados de precipitação pluviométrica $(\mathrm{mm})$ dos períodos de amostragens.

\section{RESULTADOS E DISCUSSÃO}

\section{Dinâmica da densidade larval}

Ao se quantificarem as larvas de Scarabaeidae, na cultura do milho e nabo forrageiro, em 2004, foram encontradas 0,1 larvas $\mathrm{m}^{-2}$ e 0,03 larvas $\mathrm{m}^{-2}$, respectivamente, enquanto, na cultura da soja amostrada em 2005, esta densidade foi de 3,44 larvas $\mathrm{m}^{-2}$ (Tabela 2). Ao se amostrarem as larvas novamente, nestas culturas, as mesmas tendências nas densidades larvais foram verificadas, sendo encontradas, em milho e nabo forrageiro, em 2005, e em soja, em 2006, as densidades 0,7 larvas $\mathrm{m}^{-2} ; 0,02$ larvas $\mathrm{m}^{-2}$; e 4,19 larvas $\mathrm{m}^{-2}$, respectivamente (Tabela 2).

Nas proximidades da área experimental, há predomínio de pastagens com Brachiaria decumbens Stapf e B. brizantha (Hochst. ex A. Rich.), portanto, acredita-se que as larvas observadas refiram-se às espécies de Scarabaeidae que migraram das pastagens e colonizaram a área de culturas.

Na cultura de soja, foram encontradas as maiores densidades larvais e, como esta cultura é instalada no início da estação chuvosa, provavelmente, as maiores densidades encontradas estejam relacionadas ao fato de as espécies de Scarabaeidae estarem se reproduzindo, principalmente neste período do ano.

A soja demonstra ser uma importante hospedeira, que favorece o desenvolvimento deste grupo de pragas, pois, em janeiro de 2005, a densidade amostrada foi de 3,44 larvas $\mathrm{m}^{-2}$ e, em 2006, aumentou para 4,19 larvas $\mathrm{m}^{-2}$. Assim, a combinação da época

Tabela 1. Sistemas de sucessão e épocas de semeaduras de culturas instaladas de 2006 a 2008 (Aquidauana, MS).

\begin{tabular}{|c|c|c|c|c|c|c|}
\hline \multirow{3}{*}{$\begin{array}{l}\text { Sistemas } \\
\text { de } \\
\text { sucessão }\end{array}$} & \multicolumn{6}{|c|}{ Épocas de semeaduras } \\
\hline & 2006 & \multicolumn{3}{|c|}{-2007} & \multicolumn{2}{|c|}{2008} \\
\hline & outubro & fevereiro & maio & outubro & fevereiro & maio \\
\hline 1 & Soja - CD219RR & Milho - BR 106 & Pousio & Soja - CD219RR & Milho - Minuano & Pousio \\
\hline 2 & Soja - CD219RR & Crotalaria juncea & Nabo forrageiro & Soja - CD219RR & Crotalaria juncea & Nabo forrageiro \\
\hline 3 & Soja - CD219RR & Nabo forrageiro & Pousio & Soja - CD219RR & Nabo forrageiro & Pousio \\
\hline 4 & Milho - BR 106 & Girassol - Catissol & Pousio & Milho - Minuano & Girassol - M734 & Pousio \\
\hline 5 & Milho - BR 106 & Crotalaria juncea & Nabo forrageiro & Milho - Minuano & Crotalaria juncea & Nabo forrageiro \\
\hline 6 & Milho - BR 106 & Nabo forrageiro & Pousio & Milho - Minuano & Nabo forrageiro & Pousio \\
\hline 7 & Algodão - Coodetec 405 & Milho - BR 106 & Pousio & Algodão - Delta Opal & Milho - Minuano & Pousio \\
\hline 8 & Algodão - Coodetec 405 & Crotalaria juncea & Nabo forrageiro & Algodão - Delta Opal & Crotalaria Juncea & Nabo forrageiro \\
\hline 9 & Algodão - Coodetec 405 & Nabo forrageiro & Pousio & Algodão - Delta Opal & Nabo Forrageiro & Pousio \\
\hline
\end{tabular}


Tabela 2. Densidade média $\mathrm{m}^{-2}$ (+EP) de larvas de Scarabaeidae amostradas no sistema de sucessão com soja, milho e nabo forrageiro, de maio de 2004 a janeiro de 2006, em área sob plantio direto (Aquidauana, MS).

\begin{tabular}{lc}
\hline Cultura/ Época & Densidade $\mathrm{m}^{-2}( \pm \mathrm{EP})$ \\
\hline Milho/ maio 2004 & $0,10 \pm 0,00$ \\
Nabo forrageiro/ outubro 2004 & $0,03 \pm 0,00$ \\
Soja/ janeiro 2005 & $3,44 \pm 0,33$ \\
\hline Milho/ maio 2005 & $0,70 \pm 0,00$ \\
Nabo forrageiro/ outubro 2005 & $0,02 \pm 0,00$ \\
Soja/ janeiro 2006 & $4,19 \pm 0,15$ \\
\hline
\end{tabular}

do ano com a cultura pode ser um importante fator nas densidades larvais. É possível que, para a região de Aquidauana (MS), algumas espécies de Scarabaeidae tenham se adaptado a esta cultura, o que refletiu nas maiores densidades larvais amostradas.

As menores densidades larvais foram encontradas nas amostragens realizadas em outubro de 2004 e 2005, na cultura do nabo forrageiro, o que indica que, nesta época do ano, as larvas, provavelmente, migraram no perfil do solo, buscando umidade em maiores profundidades que as amostradas, ou atingiram a fase de pupa ou adulto. A diminuição nas densidades de larvas amostradas no milho e no nabo forrageiro pode, ainda, estar associada à própria mortalidade natural que ocorre no campo, advinda da ação de patógenos ou predadores.

\section{Espécies de Scarabaeidae}

Nos sistemas de sucessão que possuem o milho semeado no verão, foram coletadas 120 larvas de Scarabaeidae, das quais foram obtidos 69 adultos, sendo $62(89,9 \%)$ da espécie L. fuscus, dois L. bidenticeps Moser e um indivíduo adulto de cada uma das seguintes espécies: Cyclocephala forsteri, C. verticalis, Geniates borelli Camerano, A. testaceipennis e Paranomala inconstans (Burmeister) (Tabela 3).

Nos sistemas de sucessão com a cultura da soja, foram coletadas 44 larvas, das quais originaram-se 28 adultos, sendo 25 (89,3\%) L. fuscus, um L. bidenticeps e dois C. forsteri. Nos sistemas de sucessão com a cultura do algodoeiro, foram coletadas 17 larvas, sendo que estas originaram $8(100 \%)$ adultos de L. fuscus (Tabela 3 ).

Nos sistemas de sucessão de culturas com soja, milho e algodoeiro, semeados no verão, a espécie predominante é L. fuscus, apresentando adaptação às várias culturas exploradas ecomomicamente. Pode-se entender que a dinâmica populacional das larvas, nos sistemas de sucessão de culturas, seja um reflexo do comportamento desta espécie.

Para a região de Aquidauana, há, portanto, predomínio das espécies de Scarabaeidae fitófagos de L. fuscus, enquanto, no Estado do Paraná, dentre as espécies ocorrentes, predomina $P$. cuyabana (Oliveira \& Garcia 2003) e, no Rio Grande do Sul, D. abderus (Silva \& Costa 2002).

Pérez-Agis et al. (2008) estudaram as espécies de larvas de Scarabaeidae presentes em cultura de milho, em duas localidades, no México, e encontraram, em San Francisco Uricho, Macrodactylus $\mathrm{sp}$, Anomala sp2, Isonychus arizonensis (Howden), Phyllophaga sp4 e Phyllophaga dentex (Bates), enquanto, em San Felipe de los Herreros, observaram Macrodactylus sp, Diplotaxis sp, Anomala punctatipennis Blanch, Anomala sp1, Phyllophaga vetula (Horn) e P. platyrhina (Bates). Aragón-Garcia \& Morón (2000) identificaram, associados à cultura de cana-de-açúcar, no México, Cyclocephala lunulata Burmeister e Phyllophaga brevidens (Bates).

Como verificado, em difrentes localidades e em cada associação de culturas, podem ser encontradas espécies distintas, justificando-se, assim, os estudos regionais, face às diferenças nos complexos de Scarabaeidae fitófagos predominantes.

\section{Efeito da sucessão de culturas na densidade de larvas}

Para as larvas amostradas em janeiro de 2007, nas culturas da safra, verifica-se que o maior número médio de larvas foi obtido na suscessão milho, girassol e pousio, a qual diferiu do sistema soja, crotalária

Tabela 3. Espécies de Scarabaeidae fitófagos amostrados em sistemas de sucessão de culturas, em área de plantio direto (Aquidauana, MS).

\begin{tabular}{lccc}
\hline \multirow{2}{*}{\multicolumn{1}{c}{ Espécies }} & \multicolumn{3}{c}{ Sistemas de sucessão } \\
\cline { 2 - 4 } & Milho & Soja & Algodão \\
\hline Liogenys fuscus & 62 & 25 & 8 \\
Liogenys bidenticeps & 2 & 1 & - \\
Cyclocephala forsteri & 1 & 2 & - \\
Cyclocephala verticalis & 1 & - & - \\
Geniates borelli & 1 & - & - \\
Anomala testaceipennis & 1 & - & - \\
Paranomala inconstans & 1 & - & - \\
\hline
\end{tabular}


e nabo forrageiro e do sistema soja, nabo e pousio, e dos três sistemas que incluem o algodoeiro (Tabela 4). As maiores densidades larvais encontradas nos três sistemas que possuem a cultura do milho na safra e o sistema que possui a soja, milho e pousio indicam que estas sucessões de culturas são mais adequadas ao desenvolvimento de L. fuscus, uma vez que estas culturas parecem fornecer alimentos mais adequados ao desenvolvimento das espécies de Scarabeideos encontradas.

Não foram observadas diferenças significativas entre as médias das densidades larvais amostradas em maio e setembro de 2007 (Tabela 4). Nos nove sistemas de sucessão, observa-se decréscimo na densidade de larvas amostradas em janeiro, maio e outubro, respectivamente (Tabela 4).

As densidades amostradas em cada época, ao serem correlacionadas com a precipitação pluviométrica, apresentaram alto coeficiente de correlação $\left(\mathrm{R}^{2}=0,85\right)(\mathrm{p}<0,05)$, demonstrando que a umidade presente no solo é um importante componente, que interfere na densidade populacional larval das espécies de Scarabaeidae ocorrentes nos sistemas de sucessão de culturas.

No segundo ano de avaliações, encontrou-se, em janeiro de 2008, novamente no sistema com milho, girassol e pousio, a maior densidade larval, diferindo, significativamente, das médias de densidades larvais dos sistemas que possuem a cultura da soja e do algodoeiro (Tabela 4). Nos sistemas com a cultura do milho, não foram encontradas diferenças significativas entre as médias de densidades larvais, comprovando que as maiores densidades larvais ocorreram nestes sistemas.

Em duas safras consecutivas, nos sistemas de sucessão com a cultura do milho, foram amostradas as maiores densidades larvais, indicando que estes sistemas permitem o desenvolvimento das larvas de Scarabaeidae, principamente de L. fuscus, as quais atingem a fase adulta e, nesta cultura, realizam suas oviposições, refletindo, assim, nas maiores densidades (Tabela 4).

Nos sistemas avaliados na safrinha e inverno, em maio e setembro de 2008, não foram verificadas diferenças estatísticas entre os sistemas de sucessão (Tabela 4). Novamente, o decréscimo de larvas foi observado na safrinha e inverno, quando comparados com a safra (Tabela 4).

Comparando-se as densidades de larvas amostradas nos sistemas que possuem a cultura da soja, verifica-se aumento de densidade no segundo ano, no sistema com soja, nabo forrageiro e pousio, sendo possível que esta combinação de sucessão de culturas esteja sendo benéfica, mais especificamente ao desenvolvimento de L. fuscus (Tabela 4). Assim, o sistema com soja, milho e pousio e com soja, crotalária e nabo forrageiro não permitiram o aumento populacional significativo de larvas, sendo estas combinações de culturas menos favoráveis ao desenvolvimento de L. fuscus.

Tabela 4. Densidade média $\mathrm{m}^{-2}( \pm$ EP) de larvas de Scarabaeidae amostradas nos diferentes sistemas de sucessão de culturas com soja, milho e algodão, em janeiro, maio e setembro de 2007 e 2008, em área sob plantio direto (Aquidauana, MS).

\begin{tabular}{|c|c|c|c|c|c|c|}
\hline \multirow{2}{*}{ Sistemas } & \multicolumn{6}{|c|}{ Densidade de larvas $\mathrm{m}^{-2}$ em épocas de amostragens } \\
\hline & jan./2007 & maio/2007 & set./2007 & jan./2008 & maio/2008 & set./2008 \\
\hline \multicolumn{7}{|l|}{ soja } \\
\hline 1 & $2,8 \pm 0,36 \mathrm{ab} A$ & $1,9 \pm 0,39$ a $\mathrm{ABC}$ & $1,0 \pm 0,12$ a C & $2,9 \pm 0,24 \mathrm{c} \mathrm{A}$ & $2,1 \pm 0,23 \mathrm{a} A \mathrm{~B}$ & $1,3 \pm 0,20$ a BC \\
\hline 2 & $2,0 \pm 0,08 \mathrm{~b} \mathrm{AB}$ & $1,4 \pm 0,27$ a B & $1,0 \pm 0,00$ a B & $2,6 \pm 0,69 \mathrm{c} A$ & $1,7 \pm 0,10 \mathrm{a} A B$ & $1,0 \pm 0,00$ a B \\
\hline 3 & $2,1 \pm 0,24 \mathrm{~b} \mathrm{~B}$ & $1,7 \pm 0,70$ a BC & $1,0 \pm 0,12$ a C & $3,5 \pm 0,30 \mathrm{bcA}$ & $1,7 \pm 0,16$ a $\mathrm{BC}$ & $1,1 \pm 0,12$ a BC \\
\hline \multicolumn{7}{|l|}{ milho } \\
\hline 4 & $3,4 \pm 0,33$ a B & $1,0 \pm 0,00$ a C & $1,0 \pm 0,00$ a C & $5,4 \pm 0,13$ a $\mathrm{A}$ & $2,0 \pm 0,30 \mathrm{a} \mathrm{C}$ & $1,0 \pm 0,00$ a $C$ \\
\hline 5 & $2,4 \pm 0,30 \mathrm{abB}$ & $1,5 \pm 0,17$ a $\mathrm{BC}$ & $1,0 \pm 0,00 \mathrm{a} \mathrm{C}$ & $4,3 \pm 0,49 \mathrm{ab} A$ & $2,1 \pm 0,19$ a B & $1,0 \pm 0,00$ a $C$ \\
\hline 6 & $2,6 \pm 0,18 \mathrm{ab} B$ & $1,2 \pm 0,14$ a CD & $1,0 \pm 0,00$ a D & $4,3 \pm 0,13 \mathrm{ab} A$ & $2,1 \pm 0,35$ a BC & $1,0 \pm 0,00$ a D \\
\hline \multicolumn{7}{|l|}{ algodão } \\
\hline 7 & $2,0 \pm 0,24 \mathrm{~b} \mathrm{AB}$ & $1,1 \pm 0,12$ a B & $1,0 \pm 0,00$ a B & $2,4 \pm 0,36 \mathrm{c} \mathrm{A}$ & $2,3 \pm 0,34$ a $A$ & $1,0 \pm 0,00$ a B \\
\hline 8 & $1,9 \pm 1,51 \mathrm{~b} \mathrm{AB}$ & $1,1 \pm 0,12$ a B & $1,0 \pm 0,00$ a B & $2,7 \pm 0,11 \mathrm{c} \mathrm{A}$ & $1,9 \pm 0,37 \mathrm{a} A B$ & $1,0 \pm 0,00$ a B \\
\hline 9 & $1,9 \pm 0,27 \mathrm{~b} \mathrm{AB}$ & $1,7 \pm 0,42 \mathrm{a} A \mathrm{~B}$ & $1,0 \pm 0,00$ a B & $2,6 \pm 0,40 \mathrm{c} \mathrm{A}$ & $2,3 \pm 0,17$ a A & $1,0 \pm 0,00$ a B \\
\hline$y=$ & & & & & & \\
\hline
\end{tabular}

Médias com letras minúsculas distintas, na coluna, e maiúsculas, na linha, diferem entre si, a 5\%. 
Para o sistema que possui soja, milho e pousio, não houve aumento, do primeiro para o segundo ano, na densidade de larvas, demonstrando que, mesmo a soja podendo permitir o desenvolvimento de imaturos de L.fuscus, outras culturas disponíveis foram menos favoráveis, o que refletiu em estagnação na densidade de larvas do primeiro para o segundo ano (Tabela 4). No sistema que possui soja, crotalária e nabo forrageiro, observou-se a mesma dinâmica populacional das larvas verificada para o sistema com soja, milho e pousio.

O efeito de sistemas de sucessão de culturas sobre a oviposição de $D$. abderus foi estudado por Silva et al. (1996), sendo verificado que sucessões com aveia (Avena sativa L.)-soja (inverno-verão) e trigo (Triticum aestivum L.)-soja (inverno-verão) favorecem a oviposição desta praga, provavelmente devido ao maior acúmulo de palhada na superfície do solo, durante o período de reprodução do inseto.

Nos três sistemas que possuem a cultura do milho semeado em outubro, ao se compararem as densidades amostradas em 2007 e 2008, encontraram-se as maiores densidades de larvas nos sistemas amostrados em 2008, sendo observadas diferenças significativas entre os anos de amostragem, o que indica que os sistemas de sucessões utilizados não promoveram redução nas densidades de larvas de L. fuscus (Tabela 4). No sistema com milho, girassol e pousio, foram verificadas as maiores densidades larvais, nos dois anos de avaliações, indicando que esta sequência de culturas é muito favorável ao desenvolvimento de L. fuscus em Aquidauana (MS).

Sistemas de sucessão envolvendo azevém, colza, ervilhaca ou tremoço com milho, sendo o milho semeado em setembro ou outubro, propiciaram menor quantidade de restos culturais sobre o solo, desfavorecendo a oviposição e diminuindo a ação de larvas de D. abderus (Silva et al. 1996). Para as sucessões utilizadas com o milho na safra, em Aquidauana (MS), não houve efeito que levasse à estagnação ou diminuição da densidade de L. fuscus.

Nos três sistemas que possuem a cultura do algodoeiro, semeada no verão, ao se analisarem as densidades de larvas amostradas em janeiro de 2007 e 2008, verifica-se que não houve diferenças estatísticas entre as médias dos anos de avaliações, demonstrando que os sistemas utilizados não permitiram aumento populacional significativo de L. fuscus (Tabela 4).
Nos sistemas de sucessão com a cultura do algodoeiro, nota-se, pelas densidades larvais, que estes sistemas interferiram no desenvolvimento de L. fuscus e nas demais espécies ocorrentes, pois, nas amostragens de 2008, foram encontradas as menores densidades, quando comparadas com os sistemas que possuem o milho (Tabela 4).

Dentre as três culturas instaladas na safra, verifica-se que o milho favoreceu o desenvolvimento deste grupo de pragas, quando comparado com a soja e o algodoeiro. A cultura do algodoeiro apresenta-se em grande expansão no Centro-Oeste brasileiro. Assim, esta cultura pode ser integrada em sistemas de rotação ou sucessão de culturas, em áreas que possuam altas populações de L. fuscus, principalmente onde se utiliza o milho na safra.

\section{CONCLUSÕES}

1. As espécies de Scarabaeidae fitófagas ocorrem em maior quantidade na safra e em menores densidades na safrinha e no inverno.

2. Dentre as espécies de Scarabaeidae amostradas nas culturas, há predomínio de L. fuscus.

3. O sistema de sucessão soja, milho e pousio, e soja, crotalária e nabo forrageiro, bem como os três sistemas de sucessão com o algodeiro, são desfavoráveis ao desenvolvimento de L. fuscus .

4. Os sistemas de sucessão com milho na safra favorecem o desenvolvimento de L. fuscus.

\section{AGRADECIMENTOS}

Ao Dr. Sérgio Ide, Dr. Fernando Zagury

Vaz-de-Mello e Dr. Miguel Angel Morón, pela identificação dos Scarabaeidae.

\section{REFERÊNCIAS}

ARAGÓN-GARCIA, A.; MORÓN, M. A. Los coleopteros Melolonthidae asociados a la rizosfera de la cana de azucar em Chietla, Puebla, México. Folia Entomológica Mexicana, Xalapa, n. 108, p. 79-94, 2000.

OLIVEIRA, C. M.; MORÓN, M. A.; FRIZZAS, M. R. Aegopsis bolboceridus (Coleoptera: Melolonthidae): an important pest on vegetables and corn in Central Brazil. Florida Entomologist, Gainesville, v. 91, n. 2, p. 324-327, 2008. 
OLIVEIRA, C. M.; MORÓN, M. A.; FRIZZAS, M. R. First record of Phyllophaga sp aff. capillata (Coleoptera: Melolonthidae) as a soybean pest in the Brazilian “Cerrado". Florida Entomologist, Gainesville, v. 90, n. 4, p. 772-775, 2007.

OLIVEIRA, L. J. et al. Ciclo biológico de Phyllophaga cuyabana (Moser) (Scarabaeidae: Melolonthinae). Anais da Sociedade Entomológica do Brasil, Londrina, v. 25, n. 3, p. 431-437, 1996.

OLIVEIRA, L. J.; GARCIA, M. A. Flight, feeding and reproductive behavior of Phyllophaga cuyabana (Moser) (Coleotera: Melolonthidae) adults. Pesquisa Agropecuária Brasileira, Brasília, DF, v. 38, n. 2, p. 179-186, 2003.

OLIVEIRA, L. J.; HOFFMANN-CAMPO, C. B.; GARCIA, M. A. Effect of soil management on the white grub population and damage in soybean. Pesquisa Agropecuária Brasileira, Brasília, DF, v. 35, n. 5, p. 887894, 2000.

PARDO-LOCARNO, L. C. et al. Structure and composition of the white grub complex (Coleoptera: Scarabaeidae) in agroecological systems of Northern Cauca, Colombia. Florida Entomologist, Gainesville, v. 88, n. 4, p. 355$363,2005$.

PÉREZ-AGIS, S. E. et al. Analisis de diversidad del complejo "gallina ciega" (Coleoptera: Melolonthidae) en dos sistemas de produccion tradicional de maiz em la region Purhepecha, Michoacan. Acta Zoológica Mexicana, Xalapa, v. 24, n. 1, p. 221-235, 2008.

RODRIGUES, S. R. et al. Aspectos biológicos de Cyclocephala verticalis Burmeister (Coleooptera: Scarabaeidae). Neotropical Entomology, Londrina, v. 39, n. 1 , p. $15-18,2010$.

RODRIGUES, S. R. et al. Ocorrência e aspectos biológicos de Anomala testaceipennis Blanchard (Coleoptera, Scarabaeidae). Revista Brasileira de Entomologia, Curitiba, v. 52, n. 1, p. 68-71, 2008a.

RODRIGUES, S. R. et al. Occurrence, biology and behavior of Liogenys fuscus Blanchard (Insecta, Coleoptera, Scarabaeidae) in Aquidauana, Mato Grosso do Sul, Brazil. Revista Brasileira de Entomologia, Curitiba, v. 52, n. 4, p. 637-640, 2008b.
SANTOS, V.; ÁVILA, C. J. Aspectos bioecológicos de Cyclocephala forsteri Endrodi, 1963 (Coleoptera: Melolonthidae) no Estado do Mato Grosso do Sul. Revista de Agricultura, Piracicaba, v. 82, n. 1, p. 28-30, 2007.

SANTOS, V.; ÁVILA, C. J. Aspectos biológicos e comportamentais de Liogenys suturalis Blanchard (Coleoptera: Melolonthidae) no Mato Grosso do Sul. Neotropical Entomology, Londrina, v. 38, n. 6, p. 734740, 2009.

SILVA, M. T. B. Níveis de controle de Diloboderus abderus (Sturm) em trigo no plantio direto. Anais da Sociedade Entomológica do Brasil, Londrina, v. 26, n. 3, p. 435-440, 1997.

SILVA, M. T. B.; COSTA, E. C. Nível de controle de Diloboderus abderus em aveia preta, linho, milho e girassol. Ciência Rural, Santa Maria, v. 32, n. 2, p. 7-12, 2002.

SILVA, M. T. B.; COSTA, E. C. Tamanho e número de unidades de amostra de solo para amostragem de larvas de Diloboderus abderus (Sturm) (Coleoptera: Melolonthidae) em plantio direto. Anais da Sociedade Entomológica do Brasil, Londrina, v. 27, n. 2, p. 193-197, 1998.

SILVA, M. T. B. et al. Influência de sistemas de manejo de solos na oviposição de Diloboderus abderus Sturm (Coleoptera: Melolonthidae). Anais da Sociedade Entomológica do Brasil, Londrina, v. 23, n. 3, p. 543548, 1994.

SILVA, M. T. B. et al. Preferência e oviposição de Diloboderus abderus (Sturm) por restos de culturas em solo com plantio direto. Anais da Sociedade Entomológica do Brasil, Londrina, v. 25, n. 1, p. 83-87, 1996.

SILVA, M. T. B.; LOECK, A. E. Ciclo evolutivo e comportamento de Dioboderus abderus Sturm (Coleoptera: Melolonthidae) em condições de plantio direto. Anais da Sociedade Entomológica do Brasil, Londrina, v. 25, n. 2, p. 329-337, 1996.

UNIVERSIDADE ESTADUAL PAULISTA “JÚLIO DE MESQUITA FILHO” (Unesp). ESTAT. Versão 2.0. Jaboticabal: Unesp, 1994. 